\title{
Supercyclicity of weighted composition operators on spaces of continuous functions
}

\author{
M.J. Beltrán-Meneu, E. Jordá and M. Murillo-Arcila
}

October 30, 2019

\begin{abstract}
Our study is focused on the dynamics of weighted composition operators defined on a locally convex space $E \hookrightarrow\left(C(X), \tau_{p}\right)$ with $X$ being a topological Hausdorff space containing at least two different points and such that the evaluations $\left\{\delta_{x}: x \in X\right\}$ are linearly independent in $E^{\prime}$. We prove, when $X$ is compact and $E$ is a Banach space containing a nowhere vanishing function, that a weighted composition operator $C_{w, \varphi}$ is never weakly supercyclic on $E$. We also prove that if the symbol $\varphi$ lies in the unit ball of $A(\mathbb{D})$, then every weighted composition operator can never be $\tau_{p}$-supercyclic neither on $C(\mathbb{D})$ nor on the disc algebra $A(\mathbb{D})$. Finally, we obtain Ansari-Bourdon type results and conditions on the spectrum for arbitrary weakly supercyclic operators, and we provide necessary conditions for a composition operator to be weakly supercyclic on the space of holomorphic functions defined in non necessarily simply connected planar domains. As a consequence, we show that no composition operator can be weakly supercyclic neither on the space of holomorphic functions on the punctured disc nor in the punctured plane.
\end{abstract}

Keywords: weighted composition operator, weak supercyclicity, disc algebra, space of holomorphic functions

Mathematics Subject Classification (2010): 47A16, 47B33, 46E15

\section{Introduction}

Troughout this paper, let $X$ denote a topological Hausdorff space containing at least two different points and let $C(X)$ be the space of continuous functions on $X$. On this space, consider the weak topology, the pointwise convergence topology $\tau_{p}$, and whenever $X$ is compact, the supremum norm topology. Let $E$ be a locally convex space continuously included in $\left(C(X), \tau_{p}\right)$ and such that the set $\left\{\delta_{x}: x \in X\right\}$ is linearly independent in $E^{\prime}$, where $\delta_{x}$ is the functional on $E$ of point evaluation at $x$. The aim of this paper is to investigate weak forms of supercyclicity of the weighted composition operator $C_{w, \varphi}$ when it is well defined in $E$. We refer to the next section for the precise notation and definitions. 
Given an operator $T$ defined on a topological vector space $(F, \tau)$, for every $n \in \mathbb{N}$ the operator $T^{n}: F \rightarrow F$ is defined as the $n$-th iterate of $T$, i.e., $T^{n}:=T \circ \cdots$ ) $\circ T$, and $T^{0}=I$. A point $f \in F$ is said to be periodic if there exists $n \in \mathbb{N}$ such that $T^{n} f=f$, and it is a fixed point if $T f=f$. We say that an operator $T$ is $\tau$-hypercyclic and $f$ is a $\tau$-hypercyclic vector for $T$ if the orbit $\operatorname{Orb}(T, f):=\left\{T^{n} f: n=0,1, \ldots\right\}$ is dense in $(F, \tau)$. If $\operatorname{Orb}(T, \operatorname{span}\{f\})=\left\{\lambda T^{n} f: \lambda \in \mathbb{C}, n=0,1, \ldots\right\}$ is dense, we say that $T$ is $\tau$-supercyclic and $f$ is a $\tau$-supercyclic vector for $T$, and if $\operatorname{span}\{\operatorname{Orb}(T, f)\}=\operatorname{span}\left\{T^{n} f: n=0,1, \ldots\right\}$ is dense, it is said to be $\tau$-cyclic and again $f$ is called a $\tau$-cyclic vector for $T$. In the case $\tau$ denotes the weak topology, the operator is said to be weakly hypercyclic (resp. weakly supercyclic or weakly cyclic), and if $\tau=\tau_{p}$, the operator is said to be pointwise hypercyclic (resp. pointwise supercyclic or pointwise cyclic). If $F$ is a separable infinite dimensional Banach or Fréchet space and $\tau$ denotes the strong topology, the operator is said to be hypercyclic (resp. supercyclic or cyclic). It is clear that for any of the aforementioned topologies, $\tau$-hypercyclicity implies $\tau$-supercyclicity, which in turn implies $\tau$-cyclicity. In the case of Banach spaces, if we mix the norm and weak topologies we first point out that weak cyclicity is equivalent to cyclicity in the norm topology because the weak closure of the convex set $\operatorname{span}\{\operatorname{Orb}(T, f)\}$ coincides with the norm closure. However, weakly hypercyclic operators are not always norm hypercyclic as it was shown by Chan and Sanders in [13, Corollary 3.3] and weakly supercyclic operators are not necessarily norm supercyclic [35, Theorem 2.3]. In the setting of Banach spaces, norm and weakly supercyclic operators share many properties such as the density of supercyclic vectors. It is well known that if $T$ is norm supercyclic then the set of all norm supercyclic vectors for $T$ is norm dense in $F$ and Sanders [35] proved that if $T$ is weakly supercyclic, the set of all weakly supercyclic vectors for $T$ is also norm dense in $F$.

For a good exposition of the subject of linear dynamics we refer the reader to the monographs by Bayart and Matheron [6] and by Grosse-Erdmann and Peris [21], and concerning composition operators we refer to the books by Cowen and MacLuer [17] and by Shapiro [37].

Ansari and Bourdon [4] showed that an isometry on an infinite dimensional Banach space cannot be norm supercyclic. However, surjective isometries can be weakly supercyclic. Sanders proved in [36, Theorem 2] that the bilateral backward shift is weakly supercyclic on $c_{0}(\mathbb{Z})$. Shkarin improved this theorem showing that in fact the bilateral backward shift operator is weakly supercyclic on $\ell_{p}(\mathbb{Z}), p>2$ [39, Theorem 1.5]. For $1 \leq p \leq 2$, Montes-Rodríguez and Shkarin [32, Theorem 6.3] and Shkarin [39, Theorem 1.5] showed that for weighted bilateral shifts, weak supercyclicity is equivalent to supercyclicity on $\ell_{p}(\mathbb{Z})$. From this together with Ansari-Bourdon's theorem, it immediately follows that an isometric weighted bilateral shift cannot be weakly supercyclic on $\ell_{p}(\mathbb{Z})$ for $1 \leq p \leq 2$. See also [6, page 253]. Besides other results about weak forms of supercyclicity, Bayart and Matheron exhibit in [5] an example of a unitary operator on a Hilbert space which is weakly supercyclic.

From this perspective, we study supercyclity on spaces of functions endowed with the weak topology and also with the pointwise convergence topology, which is in general the 
weakest natural locally convex Hausdorff topology. We discuss here the difference between both concepts showing that they are in general different by giving an example of a $\tau_{p^{-}}$ supercyclic operator which is not cyclic, hence, not (weakly) supercyclic. For surjective isometries we get some extensions of Ansari-Bourdon's theorem for important spaces of functions. For $C(X)$ when $X$ is compact, we get that a surjective isometry (which is always a weighted composition operator by the Banach-Stone theorem) is never weakly supercyclic, and for the disc algebra $A(\mathbb{D})$ we go further. No weighted composition operator on $A(\mathbb{D})$ is pointwise supercyclic.

These results on weighted composition operators on $A(\mathbb{D})$ connect with recent research about weak and strong supercyclicity of weighted composition operators on spaces of holomorphic functions. Yousefi and Rezaei [41] and Kamali et al. [27] investigated the hypercyclicity and supercyclicity of $C_{w, \varphi}$ on the space of holomorphic functions on the disc $H(\mathbb{D})$, both with respect to the compact-open topology and to its corresponding weak topology. In [9] Bès proved, among other results, that weak supercyclicity and the topologically mixing property (a dynamical concept more restrictive than hypercyclicity) are equivalent notions for $C_{w, \varphi}$ on the space of holomorphic functions on a simply connected plane domain, which in turn are satisfied if and only if the weight $w$ is zero-free and the symbol $\varphi$ is univalent and without fixed points. More recently, in [33] Moradi et al. proved that a class of weighted composition operators which contains every composition operator on some Banach spaces of analytic functions such as the disc algebra and the analytic Lipschitz space does not contain weakly supercyclic operators. For supercyclicity and weak supercyclicity of operators defined on spaces of functions with real variable, see $[7,15,31]$ and the references therein.

There is also active research focused on the connections between spectral theory of linear operators defined on Banach or locally convex spaces, the linear dynamics of the operator $T$ and the dynamics of its adjoint $T^{\prime}$. Specially relevant are the connections between the linear dynamics of $T$ and the point spectrum $\sigma_{p}\left(T^{\prime}\right)$ of its adjoint. Herrero proved in [24] that the point spectrum of the adjoint of a supercyclic operator defined on a Hilbert space consists at most of one point, and whenever it is not empty, the dimension of the subspace formed by the eigenvectors is one. Peris extended this result in [34] to supercyclic operators defined on locally convex spaces. Ansari proved in [3] that if $T$ is cyclic and the interior of $\sigma_{p}\left(T^{*}\right)$ is empty, then $T$ has a norm dense collection of cyclic vectors. The Ansari-Bourdon theorem mentioned above about the non supercyclicity of isometries on Banach spaces is a consequence of a theorem which asserts that for a supercyclic power bounded operator $T$ defined on a Banach space, the powers of the orbits of $T$ are norm convergent to 0 . Hence, immediately one gets that the powers of the adjoint $T^{*}$ are everywhere $\omega^{*}$ convergent to 0 . For recent research extending these classic results we refer to $[1,2,14]$. In the last section of the paper we use our results about weighted composition operators to get results for arbitrary operators defined on locally convex spaces, obtaining conditions in the dynamics of the adjoint $T^{\prime}$ which are necessary for $T$ being weakly supercyclic. In case of operators defined on Banach spaces, the necessary conditions are related to the point spectrum of the operator.

In [11], Bonet and Peris proved that every separable infinite dimensional Fréchet space 
admits a hypercyclic surjective operator. Grosse-Erdmann and Mortini showed in [22, Theorem 3.21] that if $U \subseteq \mathbb{C}$ is a non simply connected domain such that $\widehat{\mathbb{C}} \backslash U$ has finitely many bounded components then $H(U)$ does not support any hypercyclic composition operator. Bernal-González and Montes-Rodríguez proved in [8] that every simply connected domain supports an automorphism $\varphi$ such that $C_{\varphi}$ is not hypercyclic. In the same paper it is proved that on a non-simply connected domain with finite connected components we can find an automorphism $\varphi$ such that $C_{\varphi}$ is not supercyclic. Shapiro proved in [38] that the composition operator associated to a translation in $\mathbb{C} \backslash \mathbb{Z}$ is chaotic. As an application of our results we provide an example of a space of holomorphic functions endowed with the compact open topology which admits no weakly supercyclic composition operators. Concretely, we show that every composition operator on $H(\mathbb{D} \backslash\{0\})$ or on $H(\mathbb{C} \backslash\{0\})$ is never weakly supercyclic. This result is strongly connected with the open question proposed in [9, Problem 3].

\subsection{Notation and Outline of the Paper}

Our notation is standard. We denote by $E^{\prime}$ the dual of a locally convex space unless $E$ is a Banach space, in which case its dual is denoted by $E^{*}$. The adjoint of a continuous linear mapping $T \in L(E)$ is denoted by $T^{\prime}$ in the general case and by $T^{*}$ when $E$ is Banach. As mentioned in the introduction, our study is focused on dynamics of weighted composition operators defined on an infinite dimensional locally convex space $E \hookrightarrow\left(C(X), \tau_{p}\right)$ with $X$ being a locally compact topological Hausdorff space such that the evaluations $\left\{\delta_{x}\right.$ : $x \in X\}$ are linearly independent in $E^{\prime}$. Observe that the evaluations are always linearly independent in $\left(C(X), \tau_{p}\right)$. We consider a continuous function $w: X \rightarrow \mathbb{C}$ (the multiplier) and a continuous $\varphi: X \rightarrow X$ (the symbol) such that the weighted composition operator $C_{w, \varphi}: E \rightarrow E, f \mapsto w(f \circ \varphi)$ is well defined and continuous. The operator $C_{w, \varphi}$ combines the classical composition operator $C_{\varphi}: E \rightarrow E, f \mapsto f \circ \varphi$ with the pointwise multiplication operator $M_{w}: E \rightarrow E, f \mapsto w \cdot f$.

In [33], Moradi et al. provide sufficient conditions under which a weighted composition operator on a Banach space of analytic functions is not weakly supercyclic, and they proved that for some Banach spaces $Y$ of analytic functions on $\mathbb{D}$, the unweighted composition operator $C_{\varphi}$ is not weakly supercyclic. These spaces satisfy that every element in $Y$ has a continuous extension to the closed unit disc $\overline{\mathbb{D}}$ and for every $z$ in the boundary $\partial \mathbb{D}, \delta_{z}$ is bounded. The disc algebra

$$
A(\mathbb{D})=\left\{f \in H^{\infty}(\mathbb{D}): f \text { continuous on } \overline{\mathbb{D}}\right\},
$$

and the analytic Lipschitz spaces $\operatorname{Lip}_{\alpha}(\mathbb{D}), 0<\alpha \leq 1$,

$$
\left.\operatorname{Lip}_{\alpha}(\mathbb{D})=\{f \text { analytic in } \mathbb{D}: \mid f(z)-f(w)) \mid=O\left(|z-w|^{\alpha}\right) \text { for all } z, w \in \overline{\mathbb{D}}\right\},
$$

are examples of such spaces. As a corollary of Proposition 4 in [33] one immediately obtains the following: 
Corollary 1 Let $\varphi, w \in Y$ such that $\varphi(\mathbb{D}) \subseteq \mathbb{D}$ and let $a \in \overline{\mathbb{D}}$ be a fixed point of $\varphi$ such that $w(a) \neq 0$. If $C_{w, \varphi}$ is weakly supercyclic on $Y$, then the set

$$
\left\{\frac{\prod_{m=0}^{n} w\left(\varphi^{m}(z)\right)}{w^{n}(a)}, n \in \mathbb{N}\right\}
$$

is unbounded for every $z \in \overline{\mathbb{D}} \backslash\{a\}$.

In Section 2 we strengthen the necessary condition provided in Corollary 1 and we extend it to general locally convex spaces (see Proposition 4) instead of the concrete space of analytic functions $Y$. In Theorem 6 we use this result to prove the following, from which it immediately follows that a weighted composition operator can never be weakly supercyclic on $A(\mathbb{D})$, neither on a Banach space dense and continuoulsy embedded in it:

Theorem A Let $X$ be compact and $E$ a Banach space containing a nowhere vanishing function and satisfying $E \hookrightarrow\left(C(X),\|\|_{\infty}\right)$. A weighted composition operator $C_{w, \varphi}: E \rightarrow E$ is never weakly supercyclic.

We then restrict our study to the case where $E$ is a subspace of $C(X)$ endowed with the $\tau_{p}$ topology. More concretely, in Theorem 8 we provide sufficient conditions for the symbol $\varphi$ which ensure no $\tau_{p}$-supercyclicity of $C_{w, \varphi}$ such as the existence of a non-constant convergent orbit, the existence of stable orbits around a fixed point, or, whenever $X$ is compact, the existence of periodic (not fixed) points. Bès [9] gives a complete characterization of weak supercyclicity of $C_{w, \varphi}$ on $H(\mathbb{D})$. In particular, he shows that for any multiplier $w$ and any symbol $\varphi: \mathbb{D} \rightarrow \mathbb{D}$ with a fixed point the weighted composition operator $C_{w, \varphi}$ cannot be weakly supercyclic. From our Theorem 8 we show that assuming these conditions $C_{w, \varphi}$ is not $\tau_{p}$-supercyclic on $C(\mathbb{D})$, which easily implies Bès' result since $H(\mathbb{D})$ is densely embedded in $\left(C(\mathbb{D}), \tau_{p}\right)$.

In [33], Moradi et al. provide sufficient conditions under which a weighted composition operator on certain Banach spaces of analytic functions is not weakly supercyclic. For these spaces we prove that no further restriction in the operator than being well defined is needed to obtain that it can never be even pointwise supercyclic. Concretely, in Theorem 10 we prove:

Theorem B Let $E \hookrightarrow\left(C(\overline{\mathbb{D}}), \tau_{p}\right)$ be such that the evaluations on $\overline{\mathbb{D}}$ are linearly independent in $E^{\prime}$. If for some $\varphi \in A(\mathbb{D})$ and $w \in C(\overline{\mathbb{D}})$ the weighted composition operator $C_{w, \varphi}: E \rightarrow E$ is well defined, then it is not $\tau_{p}$-supercyclic. As a consequence, any weighted composition operator $C_{w, \varphi}$ is never $\tau_{p}$-supercyclic on the disc algebra $A(\mathbb{D})$ nor on the analytic Lipschitz spaces $\operatorname{Lip}_{\alpha}(\mathbb{D}), 0<\alpha \leq 1$.

In Section 3, we use our results on composition operators to obtain necessary conditions for an arbitrary operator $T$ defined on a locally convex space to be weakly supercyclic. These conditions are connected with the classical Ansari-Bourdon's theorem. More con- 
cretely, in Theorem 12 and Corollary 13 we get:

\section{Theorem C}

(i) Let $E$ be a locally convex space and $T: E \rightarrow E$ a continuous linear operator which is weakly supercyclic and satisfies $q \circ T \leq q$ for a continuous norm $q$ on $E$. Then $\sigma_{p}(T) \cap \partial \mathbb{D}=\emptyset$ and $\sigma_{p}\left(T^{\prime}\right) \cap \partial \mathbb{D}=\emptyset$. In particular, neither $T$ nor $T^{\prime}$ have non zero fixed points.

(ii) If $X$ is a Banach space and $T: X \rightarrow X$ is a weakly supercyclic operator, then both the point spectrum of $T$ and that of $T^{*}$ are contained in the open ball $B(0,\|T\|)$.

As an application of our results we succeed in showing that every composition operator on $H(U)$, for $U$ being the punctured disc or the punctured plane, is never weakly supercyclic, which is strongly related to [9, Problem 3$]$ :

Theorem $\mathbf{D}$ The spaces $H(\mathbb{D} \backslash\{0\})$ and $H(\mathbb{C} \backslash\{0\})$ admit no weakly supercyclic composition operators.

Finally, in the last section of our paper and motivated by the fact that in Proposition 4 only the pointwise convergence topology is needed, we study the connections between the concepts of weak supercyclicity and $\tau_{p}$-supercyclicity of weighted composition operators. We study the relation between these two concepts and cyclicity and we provide different examples that permit to separate them, i.e. there are pointwise cyclic and supercyclic operators which are not weakly supercyclic (Example 17), pointwise supercyclic operators which are not cyclic (Proposition 19) and cyclic operators which are not pointwise supercyclic (Example 20). In the last part of this section we pay special attention to the study of weighted composition operators defined on $C(\overline{\mathbb{D}})$ and $C(\partial \mathbb{D})$, where $\partial \mathbb{D}$ stands for the unit circle.

From our results, we conjecture that if $X$ is compact, $E \hookrightarrow\left(C(X), \tau_{p}\right)$ is a Banach space and the operator $C_{w, \varphi}: E \rightarrow E$ is isometric, then $C_{w, \varphi}$ is not $\tau_{p}$-supercyclic. Of course, the conjecture is not true when $X$ is only assumed to be locally compact, since [35] shows weak supercyclicity of the backward shift on $c_{0}(\mathbb{Z})$, which is an isometric composition operator.

\section{Dynamics of $C_{w, \varphi}$ on spaces of continuous functions}

In this section we study weak supercyclicity of the weighted composition operator defined on a separable infinite dimensional locally convex space of continuous functions $E \hookrightarrow$ $\left(C(X), \tau_{p}\right)$ such that all the evaluations $\left\{\delta_{x}: x \in X\right\}$ are linearly independent and we obtain important results in the setting of Banach spaces. Despite all the examples presented involve spaces in which all evaluations are linearly independent, Theorem 8 is stated in a more general context which will be needed in the next section. Our first result 
provides necessary conditions for $C_{w, \varphi}$ to be weakly (even pointwise) supercyclic. The proof is analogous to the one in [9, Proposition 2.1]. We include it here for the sake of completeness.

Proposition 2 If $C_{w, \varphi}: E \rightarrow E$ is $\tau_{p}$-supercyclic, then:

(i) $w$ is zero-free,

(ii) $\varphi$ is univalent.

Proof. (i) If $w\left(z_{0}\right)=0$ for some $z_{0} \in X$, then for any $f \in E$ we have $\operatorname{Orb}\left(C_{w, \varphi}, \operatorname{span}\{f\}\right) \subseteq$ $\operatorname{span}\{f\} \cup \operatorname{Ker}\left(\delta_{z_{0}}\right) \subsetneq E$, and hence is not dense in $E$ with respect to $\tau_{p}$, a contradiction. (ii) Suppose that $\varphi\left(z_{1}\right)=\varphi\left(z_{2}\right)$ for distinct $z_{1}, z_{2} \in X$. By $(i)$ and the fact that $\delta_{z_{1}}$ and $\delta_{z_{2}}$ are linearly independent, there exists $g \in E$ such that $g\left(z_{1}\right) \neq \frac{w\left(z_{1}\right)}{w\left(z_{2}\right)} g\left(z_{2}\right)$. Consider $f \in E$ a $\tau_{p}$-supercyclic function of $C_{w, \varphi}$. For

$$
\epsilon:=\left|g\left(z_{1}\right)-\frac{w\left(z_{1}\right)}{w\left(z_{2}\right)} g\left(z_{2}\right)\right|>0,
$$

let $\lambda \in \mathbb{C}, n \in \mathbb{N}$ such that

$$
\left|\lambda C_{w, \varphi}^{n} f-g\right|(z)<\frac{\epsilon}{4 \max \left\{\frac{\left|w\left(z_{1}\right)\right|}{\left|w\left(z_{2}\right)\right|}, 1\right\}}, \quad\left(z=z_{1}, z_{2}\right) .
$$

Observe that

$$
C_{w, \varphi}^{n} f\left(z_{1}\right)=C_{\varphi}^{n} f\left(z_{1}\right) \frac{w\left(z_{1}\right)}{w\left(z_{2}\right)} \prod_{j=0}^{n-1} C_{\varphi}^{j}(w)\left(z_{2}\right)=\frac{w\left(z_{1}\right)}{w\left(z_{2}\right)} C_{w, \varphi}^{n} f\left(z_{2}\right)
$$

then it follows that

$$
\begin{gathered}
\epsilon=\left|g\left(z_{1}\right)-\frac{w\left(z_{1}\right)}{w\left(z_{2}\right)} g\left(z_{2}\right)\right| \leq\left|g\left(z_{1}\right)-\lambda C_{w, \varphi}^{n} f\left(z_{1}\right)\right|+\left|\lambda C_{w, \varphi}^{n} f\left(z_{1}\right)-\frac{w\left(z_{1}\right)}{w\left(z_{2}\right)} g\left(z_{2}\right)\right| \leq \\
\frac{\epsilon}{4}+\left|\frac{w\left(z_{1}\right)}{w\left(z_{2}\right)}\right|\left|\lambda C_{w, \varphi}^{n} f\left(z_{2}\right)-g\left(z_{2}\right)\right| \leq \frac{\epsilon}{4}+\frac{\epsilon}{4},
\end{gathered}
$$

a contradiction. Then $\varphi$ must be univalent.

As a consequence of Proposition 2, in what follows we only consider weighted composition operators $C_{w, \varphi}$ such that $w$ is zero-free on $X$ and $\varphi$ is univalent.

Remark 3 If $C_{w, \varphi}$ is $\tau_{p}$-supercyclic on $E$, then for every $z \in X$ and every $\tau_{p}$-supercyclic function $f$ there exists $n \in \mathbb{N}$ such that $f\left(\varphi^{n}(z)\right) \neq 0$. Otherwise, $\operatorname{Orb}\left(C_{w, \varphi}, \operatorname{span}\{f \circ \varphi\}\right) \subseteq$ $\operatorname{Ker}\left(\delta_{z}\right) \subsetneq E$, and hence $\operatorname{Orb}\left(C_{w, \varphi}, \operatorname{span}\{f \circ \varphi\}\right)$ is $\tau_{p}$ nowhere dense. This is a contradiction since $f \circ \varphi$ is $\tau_{p}$-supercyclic if $f$ is so. 
The next proposition strengthens the necessary condition for weak supercyclicity provided in Corollary 1 and extends it to the space of continuous functions $E$, not necessarily analytic. We remark that we use the tool employed in [6, Proposition 1.26] and [34, Lemma $1]$ to prove that the adjoint of a supercyclic operator in a locally convex space $E$ cannot have two linearly independent eigenvectors.

Proposition 4 If $C_{w, \varphi}: E \rightarrow E$ is $\tau_{p}$-supercyclic, then

$$
\overline{\left\{\frac{\prod_{m=0}^{n-1} w\left(\varphi^{m}\left(z_{1}\right)\right) f\left(\varphi^{n}\left(z_{1}\right)\right)}{\prod_{m=0}^{n-1} w\left(\varphi^{m}\left(z_{2}\right)\right) f\left(\varphi^{n}\left(z_{2}\right)\right)}, n \in \mathbb{N}: f\left(\varphi^{n}\left(z_{2}\right)\right) \neq 0\right\}}=\mathbb{C}
$$

for every $\tau_{p}$-supercyclic function $f$ and every $z_{1} \neq z_{2} \in X$.

If in addition, $\left(\varphi^{n}\left(z_{1}\right)\right)_{n}$ and $\left(\varphi^{n}\left(z_{2}\right)\right)_{n}$ are convergent in $X$ to some (fixed) points a and $b$, respectively, then

$$
\overline{\left\{\frac{\prod_{m=0}^{n} w\left(\varphi^{m}\left(z_{1}\right)\right)}{\prod_{m=0}^{n} w\left(\varphi^{m}\left(z_{2}\right)\right)}, n \in \mathbb{N}\right\}}=\mathbb{C} .
$$

Proof. Let $z_{1}, z_{2} \in X$ be such that $z_{1} \neq z_{2}$. The mapping $F: E \rightarrow \mathbb{C}^{2}$ defined as

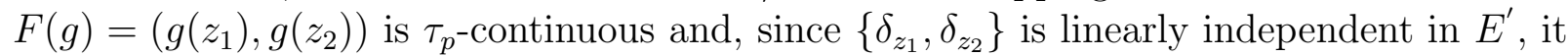
follows that $F$ is surjective. This implies that if $f \in E$ is a $\tau_{p}$-supercyclic vector of $C_{w, \varphi}$, then the set

$$
\left\{\left(\lambda \prod_{m=0}^{n-1} w\left(\varphi^{m}\left(z_{1}\right)\right) f\left(\varphi^{n}\left(z_{1}\right)\right), \lambda \prod_{m=0}^{n-1} w\left(\varphi^{m}\left(z_{2}\right)\right) f\left(\varphi^{n}\left(z_{2}\right)\right)\right): \lambda \in \mathbb{C}, n \in \mathbb{N}\right\}
$$

is dense in $\mathbb{C}^{2}$. Thus, given $c \in \mathbb{C} \backslash\{0\}$, there exists an increasing sequence $\left(n_{k}\right)_{k}$ such that $\lambda_{n_{k}} \neq 0, f\left(\varphi^{n_{k}}\left(z_{i}\right)\right) \neq 0$ for $i=1,2$, and

$$
\left(\lambda_{n_{k}} \prod_{m=0}^{n_{k}-1} w\left(\varphi^{m}\left(z_{1}\right)\right) f\left(\varphi^{n_{k}}\left(z_{1}\right)\right), \lambda_{n_{k}} \prod_{m=0}^{n_{k}-1} w\left(\varphi^{m}\left(z_{2}\right)\right) f\left(\varphi^{n_{k}}\left(z_{2}\right)\right)\right) \rightarrow(c, 1) .
$$

As a consequence,

$$
\lim _{k} \frac{\prod_{m=0}^{n_{k}-1} w\left(\varphi^{m}\left(z_{1}\right)\right) f\left(\varphi^{n_{k}}\left(z_{1}\right)\right)}{\prod_{m=0}^{n_{k}-1} w\left(\varphi^{m}\left(z_{2}\right)\right) f\left(\varphi^{n_{k}}\left(z_{2}\right)\right)}=c
$$

and the first part of the proposition holds.

If in addition, $\left(\varphi^{n}\left(z_{1}\right)\right)_{n}$ and $\left(\varphi^{n}\left(z_{2}\right)\right)_{n}$ are convergent to the fixed points $a$ and $b$ in $X$, respectively, since $\lim _{n} f\left(\varphi^{n}\left(z_{1}\right)\right)=f(a) \neq 0$ and $\lim _{n} f\left(\varphi^{n}\left(z_{2}\right)\right)=f(b) \neq 0$ by Remark 3, we get

$$
\overline{\left\{\frac{\prod_{m=0}^{n} w\left(\varphi^{m}\left(z_{1}\right)\right)}{\prod_{m=0}^{n} w\left(\varphi^{m}\left(z_{2}\right)\right)}, n \in \mathbb{N}\right\}}=\mathbb{C} .
$$


Remark 5 Observe that in Proposition 4 we do not need all evaluations $\delta_{z}, z \in X$, to be linearly independent. It is enough to assume that $\left\{\delta_{z_{1}}, \delta_{z_{2}}\right\}$ is linearly independent. Note that $w(z)$ can be zero if $\delta_{z}=0$ in $E^{\prime}$, but if $\delta_{z} \neq 0$, then $w\left(\varphi^{n}(z)\right) \neq 0$ for every $n \in \mathbb{N}_{0}$. Just consider an increasing sequence $\left(n_{k}\right)_{k}$ such that $\lambda_{n_{k}} \prod_{m=0}^{n_{k}-1} w\left(\varphi^{m}(z)\right) f\left(\varphi^{n_{k}}(z)\right)$ tends to 1 .

Now we present one of the main results of this section, which solves in the negative the problem of weak supercyclicity of the weighted composition operator on $E$ whenever $X$ is compact.

Theorem 6 Let $X$ be compact and let $E$ be a Banach space satisfying $E \hookrightarrow\left(C(X),\|\|_{\infty}\right)$ and containing a nowhere vanishing function. The weighted composition operator $C_{w, \varphi}$ : $E \rightarrow E$ is never weakly supercyclic.

Proof. If $C_{w, \varphi}: E \rightarrow E$ is weakly supercyclic, the set of weakly supercyclic vectors is norm dense in $E$, and thus, \|\|$_{\infty}$-dense [35, Proposition 2.1]. Then, by the hypothesis of existence of nowhere vanishing functions on $E$ we can easily get a weakly supercyclic function $f$ such that $\epsilon \leq|f(x)|$ for every $x \in X$ and for some $\epsilon>0$. Since the multiplication operator $M_{w}: E \rightarrow E$ is not weakly supercyclic by [6, Proposition 1.26] because $\left\{\delta_{x}, x \in X\right\}$ is a set of independent eigenvectors of $E^{*}$, we can assume that there exists some $x_{0} \in X$ such that $x_{1}=\varphi\left(x_{0}\right) \neq x_{0}$. Therefore, as $X$ is compact and $w$ and $f$ are continuous and have no zeros in $X$, there exists $C>0$ such that

$$
\left|\frac{\prod_{m=0}^{n-1} w\left(\varphi^{m}\left(x_{1}\right)\right) f\left(\varphi^{n}\left(x_{1}\right)\right)}{\prod_{m=0}^{n-1} w\left(\varphi^{m}\left(x_{0}\right)\right) f\left(\varphi^{n}\left(x_{0}\right)\right)}\right|=\left|\frac{w\left(\varphi^{n}\left(x_{0}\right)\right) f\left(\varphi^{n+1}\left(x_{0}\right)\right)}{w\left(x_{0}\right) f\left(\varphi^{n}\left(x_{0}\right)\right)}\right| \leq C
$$

for every $n \in \mathbb{N}$, a contradiction by Proposition 4 .

Our main results apply to $C(X)$ when $X$ is compact, but relaxing this hypothesis and not requiring all evaluations $\delta_{z}, z \in X$, to be linearly independent in Theorem 8, permits us to obtain more general results which are useful in the following section. We give sufficient conditions that ensure the operator $C_{w, \varphi}$ is not $\tau_{p}$-supercyclic. We first introduce the following definition.

Definition 7 Let $\varphi: X \rightarrow X$ and let $z_{0} \in X$ be a fixed point of $\varphi \cdot \varphi$ is said to have stable orbits around $z_{0}$ if there exists a fundamental family of connected compact neighbourhoods of $z_{0},\left(V_{j}\right)_{j} \subseteq X$ such that $\varphi\left(V_{j}\right) \subseteq V_{j}$ for every $j \in \mathbb{N}$.

Theorem 8 Let $X$ be a topological Hausdorff space and let $E \hookrightarrow\left(C(X), \tau_{p}\right)$. If any of the following conditions holds, then the operator $C_{w, \varphi}: E \rightarrow E$ is not $\tau_{p}$-supercyclic:

i) $\varphi$ has two fixed points $\left\{z_{1}, z_{2}\right\}$ such that the evaluations $\left\{\delta_{z_{1}}, \delta_{z_{2}}\right\} \subseteq E^{\prime}$ are linearly independent.

ii) there exists an orbit $\left\{\varphi^{n}\left(z_{1}\right), n=0,1, \ldots\right\}, z_{1} \in X$, non-constant and convergent to an element $z_{0} \in X$ satisfying $\delta_{z_{0}} \neq 0$ and $\left\{\delta_{z_{1}}, \delta_{\varphi\left(z_{1}\right)}\right\}$ linearly independent in $E^{\prime}$. 
iii) $\varphi$ has a periodic (not fixed) point $z_{1}$ satisfying that $\left\{\delta_{z_{1}}, \delta_{\varphi\left(z_{1}\right)}\right\}$ are linearly independent in $E^{\prime}$.

iv) $X$ is compact, $\varphi$ has a fixed point $z_{2}$ such that $|w(z)| \leq\left|w\left(z_{2}\right)\right|$ for all $z \in X$ and there exists $z_{1} \neq z_{2}$ such that $\left\{\delta_{z_{1}}, \delta_{z_{2}}\right\}$ are linearly independent in $E^{\prime}$.

v) All evaluations $\delta_{z}, z \in X$, are linearly independent, $\varphi$ has a fixed point $z_{0}$ such that $z_{0}$ is an accumulation point of $X$, and $\varphi$ has stable orbits around $z_{0}$.

Proof. Assume $C_{w, \varphi}$ is $\tau_{p}$-supercyclic and $f \in E$ is a $\tau_{p}$-supercyclic function. For $z_{1}$ and $z_{2}$ as in (i) the set $\left\{\left(\frac{w\left(z_{1}\right)}{w\left(z_{2}\right)}\right)^{n} \frac{f\left(z_{1}\right)}{f\left(z_{2}\right)}: n \in \mathbb{N}\right\}$ cannot be dense in $\mathbb{C}$, since it converges to 0 , diverges to infinity or lies in $r \partial \mathbb{D}$ for $r=\frac{f\left(z_{1}\right)}{f\left(z_{2}\right)}$. Applying Proposition 4 and Remark 5, we get a contradiction.

(ii) Assume $z_{2}=\varphi\left(z_{1}\right) \neq z_{1}$ and let $z_{0}=\lim _{n} \varphi^{n}\left(z_{1}\right)$. Then, $\lim _{n} \frac{\prod_{m=0}^{n-1} w\left(\varphi^{m}\left(z_{1}\right)\right) f \circ \varphi^{n}\left(z_{1}\right)}{\prod_{m=0}^{n-1} w\left(\varphi^{m}\left(z_{2}\right)\right) f \circ \varphi^{n}\left(z_{2}\right)}=$ $\frac{w\left(z_{1}\right)}{w\left(z_{0}\right)}$. Applying Proposition 4 and Remark 5, we get that $C_{w, \varphi}$ is not $\tau_{p^{-}}$supercyclic.

(iii) From the hypothesis we can get a periodic point $z_{1} \in X$ of $\varphi$ and consider $z_{2}=$ $\varphi\left(z_{1}\right)$. By Remark 3 there is $j \in \mathbb{N}$ such that $f\left(\varphi^{j}\left(z_{1}\right)\right) \neq 0$ and also there exists $C_{1}=$ $\max _{n \in \mathbb{N}}\left|f\left(\varphi^{n}\left(z_{1}\right)\right)\right| \max _{n \in \mathbb{N}}\left|w\left(\varphi^{n}\left(z_{1}\right)\right)\right|$ and $C_{2}=\min _{\left\{n \in \mathbb{N}: f\left(\varphi^{n}\left(z_{1}\right)\right) \neq 0\right\}}\left|f\left(\varphi^{n}\left(z_{1}\right)\right)\right|>0$ satisfying:

$$
\left|\frac{\prod_{m=0}^{n-1} w\left(\varphi^{m}\left(z_{2}\right)\right) f\left(\varphi^{n}\left(z_{2}\right)\right)}{\prod_{m=0}^{n-1} w\left(\varphi^{m}\left(z_{1}\right)\right) f\left(\varphi^{n}\left(z_{1}\right)\right)}\right|=\left|\frac{w\left(\varphi^{n}\left(z_{1}\right)\right) f\left(\varphi^{n+1}\left(z_{1}\right)\right)}{w\left(z_{1}\right) f\left(\varphi^{n}\left(z_{1}\right)\right)}\right| \leq \frac{C_{1}}{C_{2}\left|w\left(z_{1}\right)\right|}
$$

for all $n \in \mathbb{N}$ such that the quotients are well defined. Applying again Proposition 4 and Remark 5, we get the contradiction

(iv) By Remark 3, $f\left(z_{2}\right) \neq 0$. Also from $\delta_{z_{2}} \neq 0$ in $E^{\prime}$ we get $w\left(z_{2}\right) \neq 0$. Let $z_{1} \in X$ be as in the hypothesis. Given $M=\max \{|f(z)|: z \in X\}$, we have

$$
\left|\frac{\prod_{m=0}^{n-1} w\left(\varphi^{m}\left(z_{1}\right)\right) f\left(\varphi^{n}\left(z_{1}\right)\right)}{w\left(z_{2}\right)^{n} f\left(z_{2}\right)}\right| \leq \frac{M}{\left|f\left(z_{2}\right)\right|}, \text { for all } n \in \mathbb{N}, z \in X .
$$

Applying Proposition 4 and Remark 5 we get that $C_{w, \varphi}$ is not $\tau_{p^{-}}$supercyclic.

(v) By hypothesis and Remark 3, there exists a compact neighbourhood $V$ of $z_{0}$ such that $\varphi(V) \subseteq V$ and $f(v) \neq 0$ for all $v \in V$. Let $z_{1} \in V \backslash\left\{z_{0}\right\}$ and assume $z_{2}=\varphi\left(z_{1}\right) \neq z_{1}$ (otherwise, apply (i)). It follows that

$$
\left|\frac{\prod_{m=0}^{n-1} w\left(\varphi^{m}\left(z_{1}\right)\right) f\left(\varphi^{n}\left(z_{1}\right)\right)}{\prod_{m=0}^{n-1} w\left(\varphi^{m}\left(z_{2}\right)\right) f\left(\varphi^{n}\left(z_{2}\right)\right)}\right|=\left|\frac{w\left(z_{1}\right) f\left(\varphi^{n}\left(z_{1}\right)\right)}{w\left(\varphi^{n}\left(z_{1}\right)\right) f\left(\varphi^{n+1}\left(z_{1}\right)\right)}\right| \leq \frac{M_{1} M_{2}}{M_{3}} \text {, for all } n \in \mathbb{N} \text {, }
$$

where $M_{1}=\frac{\max \{|w(z)|: z \in V\}}{\min \{|w(z)|: z \in V\}}, M_{2}=\max \{|f(z)|: z \in V\}$ and $M_{3}=\min \{|f(v)|: v \in V\}$. From Proposition $4, C_{w, \varphi}$ cannot be $\tau_{p}$-supercyclic.

Remark 9 As a consequence of (ii) or $(\mathrm{v})$, for $E=H(\mathbb{D})$ the weighted composition operator is not $\tau_{p}$-supercyclic if $\varphi$ has a fixed point in $\mathbb{D}$. Even more, if $\varphi: \mathbb{D} \rightarrow \mathbb{D}$ is holomorphic and has a fixed point then $C_{w, \varphi}$ is not pointwise supercyclic on $\left(C(\mathbb{D}), \tau_{p}\right)$. 
In [33, Corollary 10] the authors show that the composition operator on the spaces $A(\mathbb{D})$ and $\operatorname{Lip}_{\alpha}(\mathbb{D})$ is never weakly supercyclic. In the next theorem we prove that $\tau$ supercyclicity on these spaces is not possible even for a general weighted composition $C_{w, \varphi}$ and with respect to weaker topologies.

Theorem 10 If $X=\overline{\mathbb{D}}$ and $\varphi \in A(\mathbb{D})$, then the weighted composition operator $C_{w, \varphi}$ : $E \rightarrow E$ is never $\tau_{p}$-supercyclic. As a consequence, $C_{w, \varphi}$ is never $\tau_{p}$-supercyclic on the disc algebra $A(\mathbb{D})$ and the analytic Lipschitz spaces Lip $(\mathbb{D}), 0<\alpha \leq 1$.

Proof. By the Denjoy-Wolff theorem [12, Theorem 0.2], if $\varphi$ is not the identity and not an automorphism with exactly one fixed point, then there is a unique (fixed) point $z_{0} \in \overline{\mathbb{D}}$ such that $\left(\varphi^{n}\right)_{n}$ converges to $z_{0}$ uniformly on the compact subsets of $\mathbb{D}$. Theorem 8 (ii) and (v) cover all the possible cases for $\varphi$, therefore $C_{w, \varphi}$ is never $\tau_{p}$-supercyclic.

\section{Weak supercyclicity on Fréchet spaces}

Ansari and Bourdon proved in [4] that if $X$ is a Banach space and $T: X \rightarrow X$ is a power bounded and supercyclic operator, then $\left(T^{n}(x)\right)_{n}$ converges to 0 for each $x \in X$. From this result it follows that isometries in Banach spaces are never supercyclic. Albanese and Jornet [1] have recently extended this result for operators in locally convex spaces. Concretely, they show that if $E$ is a locally convex space and $T: E \rightarrow E$ is a supercyclic operator such that $\left(T^{n}\right)_{n}$ is an equicontinuous sequence in $L(E)$, then $\left(T^{n}(e)\right)_{n}$ converges to 0 for any $e \in E$. In particular, this property applies to barrelled spaces, where the condition of equicontinuity of $\left(T^{n}\right)_{n}$ is equivalent to the boundedness of the sequence $\left(T^{n}(e)\right)_{n}$ in $E$ for any $e \in E$. As an application of Theorem 8 we get below Ansari-Bourdon type results for weakly supercyclic operators.

Theorem 11 Let $E$ be a locally convex space and let $T: E \rightarrow E$ be a continuous linear weakly supercyclic operator. If $u \in E^{\prime}$ is not a fixed point of $T^{\prime}$ and it satisfies that $\left(T^{\prime n}(u)\right)_{n}$ is weakly convergent to some (fixed point) $v \in E^{\prime}$, then $v=0$.

Proof. Consider in $E^{\prime}$ the weak-star topology $\omega^{*}$. Observe that $(E, \omega)$ can be identified as a subspace of $\left(C\left(E^{\prime}\right), \tau_{p}\right)$ by means of $e(u)=\langle u, e\rangle$ for every $e \in E$ and $u \in E^{\prime}$. With this identification, $T=C_{\varphi}$ for $\varphi=T^{\prime}$, as $C_{T^{\prime}}(e)(u)=\left(e \circ T^{\prime}\right)(u)=\left\langle e, T^{\prime} u\right\rangle=\langle T e, u\rangle$ for every $u \in E^{\prime}$. Now the result is a direct consequence of Theorem 8 (ii). Observe that in the case $u$ and $T^{\prime}(u)$ are not linearly independent, if $\left(T^{\prime n}(u)\right)_{n}=\left(\lambda^{n} u\right)_{n}, \lambda \in \mathbb{C}$, is weakly convergent to some $v$, then $v=0$.

Assuming some equicontinuity conditions on the operator with respect to the strong topology, the last theorem can be improved:

Theorem 12 Let $E$ be a locally convex space and $T: E \rightarrow E$ a continuous linear operator which is weakly supercyclic and satisfies $q \circ T \leq q$ for a continuous norm $q$ of $E$. Then 
$\sigma_{p}(T) \cap \partial \mathbb{D}=\emptyset$ and $\sigma_{p}\left(T^{\prime}\right) \cap \partial \mathbb{D}=\emptyset$. In particular, neither $T$ nor $T^{\prime}$ have non zero fixed points.

Proof. Since $T$ is weakly supercyclic if and only if $\alpha T$ is so for any $\alpha \in \partial \mathbb{D}$, we only need to show $1 \notin \sigma_{p}(T)$ and $1 \notin \sigma_{p}\left(T^{\prime}\right)$. Let $U=\{e \in E: q(e) \leq 1\}$ and consider $K:=\left(U^{\circ}, \omega^{*}\right)$, which is a compact space by the Alaoglu Bourbaki theorem. Notice that $U^{\circ}:=\left\{u \in E^{\prime}:|u(e)| \leq 1\right.$ for all $\left.e \in U\right\}=\left\{u \in E^{\prime}:|u(e)| \leq q(e)\right.$ for all $\left.e \in E\right\}$ and from the hypothesis $q \circ T \leq q$ it follows that $T^{\prime}\left(U^{\circ}\right) \subseteq U^{\circ}$. There is a continuous injection $i:(E, \omega) \hookrightarrow\left(C(K), \tau_{p}\right)$. Under identification of $E$ with the corresponding subspace of $\left(C(K), \tau_{p}\right)$ the operator $T$ is the composition operator $C_{\varphi}$ where $\varphi=T^{\prime}$.

The assertion $1 \notin \sigma_{p}\left(T^{\prime}\right)$, which is equivalent to saying that $\varphi$ does not have any fixed point, follows now from Theorem 8 (iv). Let us now see that $1 \notin \sigma_{p}(T)$. Assume that $T\left(e_{0}\right)=e_{0}$ for some $e_{0} \in U, q\left(e_{0}\right)=1$, and let $F\left(e_{0}\right):=\left\{u \in U^{\circ}: u\left(e_{0}\right)=1\right\}$. Observe that $T^{\prime}\left(F\left(e_{0}\right)\right) \subseteq F\left(e_{0}\right)$ and from the Hahn Banach theorem, $F\left(e_{0}\right)$ is nonempty. Moreover, it is a $\omega^{*}$-compact convex set. From Schauder-Tychonoff's fixed point theorem [28, Page 230], we get a fixed point for $T^{\prime}$ and we conclude.

From Theorem 12 and the fact that an operator $T$ is weakly supercyclic if and only if $a T$ is so for each $a \in \mathbb{C}$, it easily follows the next consequence:

Corollary 13 Let $X$ be a Banach space. If $T: X \rightarrow X$ is a weakly supercyclic operator, then $\sigma_{p}(T) \subseteq B(0,\|T\|)$ and $\sigma_{p}\left(T^{*}\right) \subseteq B(0,\|T\|)$, where $B(0,\|T\|)$ stands for the open disc of radius $\|T\|$ centered at zero.

We remark that the assertion for $T^{*}$ in the above corollary can also be obtained from [6, Proposition 1.26]. In fact, if $\alpha \in \sigma_{p}\left(T^{*}\right)$ then from [6, Proposition 1.26] it follows that $\sigma_{p}\left(T^{*}\right)=\{\alpha\}$ and $(1 / \alpha) T$ restricted to an invariant closed hyperplane $X_{0}$ of $X$ is weakly hypercyclic. Hence, $|\alpha|<\left\|\left.T\right|_{X_{0}}\right\| \leq\|T\|=\left\|T^{*}\right\|$. For the special case of operators of the form $\lambda I \oplus T: \mathbb{C} \oplus X \rightarrow \mathbb{C} \oplus X$, weak supercyclicity implies weak hypercyclicity of $(1 / \lambda) T$ in $X$ by [35, Theorem 2.2], and hence also the inequality $|\lambda|<\|T\| \leq\|\lambda I \oplus T\|$.

We can now give a corollary related to [9, Theorem 3.1]. Given a simply connected domain $U$, the operator $C_{\varphi}$ is hypercyclic on $H(U)$ if and only if it is weakly supercyclic. Moreover, it is equivalent to the absence of fixed points for $\varphi$ and its injectivity. In case $U=\mathbb{D}$ this is equivalent by the Denjoy-Wolff theorem to $\varphi$ being strongly runaway, that is, for each $K \subset \mathbb{D}$ compact there is $n_{0}$ such that $\varphi^{n}(K) \cap K=\emptyset$ for each $n \geq n_{0}$. This equivalence is extended by Kalmes [26, Theorem 6.2] to composition operators on kernels $C_{P}^{\infty}(X)$ of constant coefficient elliptic differential operators with $X \subseteq \mathbb{R}^{d}$ open and homeomorphic to $\mathbb{R}^{d}$. Our next result involves hyperbolic domains. For the definition of a hyperbolic domain $U \subseteq \mathbb{C}$ we refer to [30], where it is given in a more general context. This notion includes all complex domains except $\mathbb{C}$ and $\mathbb{C} \backslash\{z\}$, for $z \in \mathbb{C}$ [30, Lemma 2.5]. Corollary 14 below could be stated for a general hyperbolic domain $U$ as contemplated in [30].

Corollary 14 Let $U \subseteq \mathbb{C}$ be a hyperbolic domain and let $\varphi: U \rightarrow U$ be holomorphic. If $C_{\varphi}: H(U) \rightarrow H(U)$ is weakly supercyclic then $\varphi$ is injective and strongly runaway. 
Proof. The injectivity of the symbol $\varphi$ was obtained by Bès in [9, Proposition 2.1]. By [30, Theorem 5.2], we have four possible cases for $\varphi$. If $\varphi$ has a fixed point, $C_{\varphi}$ is not weakly supercyclic by [9, Proposition 2.1]. If there exists $n_{0}$ such that $\varphi^{n_{0}}=I d_{U}, C_{\varphi}$ is certainly not weakly supercyclic. If there exists a compact subset $K$ containing an accumulation point such that $\varphi(K) \subseteq K$, then $C_{\varphi}$ is not weakly supercyclic applying Theorem 12, since the constant functions are fixed points of $C_{\varphi}$ and $p_{K}(f)=\{\sup |f(z)|: z \in K\}, f \in H(U)$, is a continuous norm in $H(U)$. Thus, the only remaining possibility is that $\varphi$ is strongly runaway.

We finish this section with an application of our results strongly connected with the open question proposed in [9, Problem 3]. For $U$ being the punctured disc or the punctured plane, we succeed in showing that every composition operator on $H(U)$ is never weakly supercyclic.

Theorem 15 The spaces $H(\mathbb{D} \backslash\{0\})$ and $H(\mathbb{C} \backslash\{0\})$ admit no weakly supercyclic composition operators.

Proof. We prove first the case of the punctured disc. If $\varphi$ is a self-map on $\mathbb{D} \backslash\{0\}$ then $\varphi$ admits a holomorphic extension $\hat{\varphi}: \mathbb{D} \rightarrow \mathbb{D}$. If $\hat{\varphi}(0) \neq 0$, then $f \circ \varphi$ admits a holomorphic extension to $\{0\}$ for each $f \in H(\mathbb{D} \backslash\{0\})$. Since $H(\mathbb{D})$ is closed in $H(\mathbb{D} \backslash\{0\})$, we get that $C_{\varphi}$ is not weakly supercyclic.

Assume now that $\widehat{\varphi}(0)=0$ and that $C_{\varphi}$ is weakly supercyclic. By Corollary $14, \varphi$ is strongly runaway, and thus, $\widehat{\varphi}$ is not an elliptic automorphism. Also by Corollary 14 we deduce that $\widehat{\varphi}$ is injective, and so, $\widehat{\varphi}^{\prime}(z) \neq 0$ for every $z \in \mathbb{D}$. Then, by Koenig's theorem [30, Theorem 8.2] there is $0<r<1$ and a conformal (holomorphic and injective) mapping $F: D(0, r) \rightarrow \mathbb{C}$ with $F(0)=0$ and $0<|a|<1$ such that

$$
g_{a}=F \circ \widehat{\varphi} \circ F^{-1},
$$

where $g_{a}(z)=a z$ and, since $\widehat{\varphi}(D(0, r)) \subseteq D(0, r)$ by Schwarz's lemma, for $U:=F(D(0, r))$ we get $g_{a}(U) \subseteq U$. From $F(0)=0$ and $F$ injective, we get that $F$ maps conformally $D(0, r) \backslash\{0\}$ to $U \backslash\{0\}$, and hence $H(U \backslash\{0\})$ is isomorphic to $H(D(0, r) \backslash\{0\})$ by means of $\left.f \mapsto f \circ F\right|_{D(0, r) \backslash\{0\}}$. As $\widehat{\varphi}(D(0, r)) \subseteq D(0, r)$ and $\widehat{\varphi}$ is injective, $\varphi(D(0, r) \backslash\{0\}) \subseteq$ $D(0, r) \backslash\{0\}$. This implies that $C_{\left.\varphi\right|_{D(0, r) \backslash\{0\}}}$ is weakly supercyclic on $H(D(0, r) \backslash\{0\})$ if and only if $C_{g_{a}}$ is weakly supercyclic in $H(U \backslash\{0\})$ because they are two conjugated systems. Moreover, the restriction mapping $r: H(\mathbb{D} \backslash\{0\}) \rightarrow H(D(0, r) \backslash\{0\}), f \mapsto f_{\mid}$, has dense range by Runge's theorem. Therefore, we only need to show that $C_{g_{a}}$ is not weakly supercyclic in $H(U \backslash\{0\})$.

Since $F$ is conformal we get that $U=F(D(0, r))$ is simply connected. Let $s>0$ such that the circle $C_{s}$ of radius $s$ is contained in $U$. The projections $\left(P_{k}\right)_{k \in \mathbb{Z}}$ on the Laurent development in $H(U \backslash\{0\})$ are continuous functionals, and

$$
P_{k}\left(f \circ g_{a}\right)=\frac{1}{2 \pi i} \int_{C_{s}} z^{-k-1} f(a z) d z=a^{k} P_{k}(f),
$$


for each $k \in \mathbb{Z}$. Hence, if $f$ is a weakly supercyclic vector of $C_{g_{a}}$ then $P_{k}(f) \neq 0$ for each $k \in \mathbb{Z}$. We assume without loss of generality $P_{0}(f)=1$. If $\lim _{i} \lambda_{i}\left(f \circ\left(g_{a}\right)^{n_{i}}\right)=1, n_{i} \geq 1$ for all $i$, we get

$$
\lim _{i} \lambda_{i}=\lim _{i} P_{0}\left(\lambda_{i}\left(f \circ\left(g_{a}\right)^{n_{i}}\right)\right)=P_{0}(1)=1
$$

But we also have

$$
0=\left|P_{-1}(1)\right|=\lim _{i}\left|P_{-1}\left(\lambda_{i}\left(f \circ\left(g_{a}\right)^{n_{i}}\right)\right)\right| \geq \lim _{i}\left|\lambda_{i}\right|\left|P_{-1}(f)\right| \liminf _{i}\left|a^{-n_{i}}\right| \geq\left|\frac{P_{-1}(f)}{a}\right|,
$$

a contradiction.

Assume now that $C_{\varphi}: H(\mathbb{C} \backslash\{0\}) \rightarrow H(\mathbb{C} \backslash\{0\})$ is weakly supercyclic. Then $\varphi$ is an injective holomorphic self map on $\mathbb{C} \backslash\{0\}$. An injective self map $\varphi$ on $\mathbb{C} \backslash\{0\}$ has the form $\varphi(z)=a z$ or $\varphi(z)=\frac{a}{z}$, with $a \in \mathbb{C} \backslash\{0\}$ (see [18, Theorem 25.3.1]). The case $\varphi(z)=\frac{a}{z}$ follows immediatly since $C_{\varphi}^{2}=I d$. We then consider the case $\varphi(z)=a z$. If $|a| \leq 1$ we proceed as in the punctured disc. If $|a|>1$ then we proceed as in the punctured disc but getting a contradiction with the projection $P_{1}(f)$.

Grosse-Erdmann and Mortini proved in [16, Proposition 2.3] that symbols of hypercyclic composition operators defined on $H(U)$ for $U$ being a domain in $\mathbb{C}$ are always runaway. We finish the section seeing that the statement of Grosse-Erdmann and Mortini remains true if we replace hypercyclic by weakly supercyclic.

Proposition 16 Let $U \subseteq \mathbb{C}$ be a domain and $\varphi: U \rightarrow U$ a holomorphic mapping. If $C_{\varphi}: H(U) \rightarrow H(U)$ is weakly supercyclic then $\varphi$ is strongly runaway.

Proof. If $U=\mathbb{C}$, apply [9, Theorem 3.1] and [16, Proposition 2.3]. If $U$ is hyperbolic then the result follows from Corollary 14. The remaining case is a punctured plane by $[30$, Lemma 2.5], and thus it follows from Theorem 15.

\section{Remarks on the dynamics of $C_{w, \varphi}$ with respect to the pointwise topology}

In this section, we focus on the following problem: given a topological Hausdorff space $X$, can $C_{w, \varphi}: C(X) \rightarrow C(X)$ be pointwise supercyclic? We answer this question in the positive for certain sequence spaces. Moreover, we study the connections between the concepts of weak supercyclicity, pointwise supercyclicity and cyclicity. It is clear that weak supercyclicity implies $\tau_{p}$-supercyclicity and cyclicity, since cyclicity in the weak topology is equivalent to cyclicity with respect to the norm topology. However, we show that there exist $\tau_{p}$-supercyclic operators which are not weakly supercyclic, and that the concepts of $\tau_{p}$-supercyclicity and cyclicity are not related. We illustrate these concepts and connections with several examples. 
Given $X$ a locally compact space, we denote by $\hat{X}:=X \cup\{\infty\}$ the Alexandroff compactification of $X$. Any homeomorphism $\varphi: X \rightarrow X$ extends uniquely to $\hat{\varphi}: \hat{X} \rightarrow \hat{X}$, $\hat{\varphi}(\infty):=\infty$ and $\hat{\varphi}_{\mid X}=\varphi$.

For $X=\mathbb{Z}, C(\mathbb{Z})$ is the set of bilateral sequences $\left\{f=\left(f_{n}\right)_{n=-\infty}^{\infty}: f_{n} \in \mathbb{C}, n \in \mathbb{Z}\right\}$. Observe that $C(\hat{\mathbb{Z}})$ is the Banach space

$$
c_{\infty}(\mathbb{Z})=\left\{f=\left(f_{n}\right)_{n=-\infty}^{\infty}, f_{n} \in \mathbb{C}, n \in \mathbb{Z}: f_{n} \text { is convergent as }|n| \rightarrow \infty\right\},
$$

endowed with the supremum norm $\|f\|_{\infty}=\sup _{n \in \mathbb{Z}}\left|f_{n}\right|$. Indeed, a bilateral sequence is a continuous map $f: \mathbb{Z} \rightarrow \mathbb{C}$, and it converges if and only if this map has an extension to a continuous map $\hat{f}: \hat{\mathbb{Z}} \rightarrow \mathbb{C}$, where the basic-open neighbourhoods of $\infty$ are cofinite. The value at infinity is the limit of the sequence.

Consider now a particular case of composition operators. For each $j \in \mathbb{Z}$, let $e_{j}$ denote the bilateral sequence $(\ldots, 0,1,0, \ldots)$ with the 1 in the $j$-th position and consider the bilateral backward shift $B: c_{\infty}(\mathbb{Z}) \rightarrow c_{\infty}(\mathbb{Z})$ defined by $B e_{j}=e_{j-1}$ for each $j \in \mathbb{Z}$. $B$ is the composition operator $C_{\varphi}: c_{\infty}(\mathbb{Z}) \rightarrow c_{\infty}(\mathbb{Z})$ associated to the symbol $\varphi: \mathbb{Z} \rightarrow \mathbb{Z}, j \mapsto j+1$. It is well known that $B: c_{0}(\mathbb{Z}) \rightarrow c_{0}(\mathbb{Z})$ is a weakly supercyclic isometry [36, Theorem 2], where

$$
c_{0}(\mathbb{Z})=\left\{f=\left(f_{n}\right)_{n=-\infty}^{\infty} \in c_{\infty}(\mathbb{Z}): \lim _{|n| \rightarrow \infty} f_{n}=0\right\} .
$$

However, in the next example we show that $B: c_{\infty}(\mathbb{Z}) \rightarrow c_{\infty}(\mathbb{Z})$ is not weakly supercyclic, but it is $\tau_{p}$-supercyclic. As a consequence, we get that these weak forms of supercyclicity are not equivalent for composition operators.

Example 17 The bilateral backward shift $B: c_{\infty}(\mathbb{Z}) \rightarrow c_{\infty}(\mathbb{Z})$ is not weakly supercyclic but it is $\tau_{p}$-supercyclic. Indeed, as $B$ is weakly supercyclic on $c_{0}(\mathbb{Z})$ [36, Theorem 2], it is also $\tau_{p}$-supercyclic. Since $c_{0}(\mathbb{Z})$ is dense in $c_{\infty}(\mathbb{Z})$ with respect to the pointwise convergence topology on $\mathbb{Z}$, we get that $B$ is $\tau_{p}$-supercyclic on $c_{\infty}(\mathbb{Z})$. However, $B$ is not weakly supercyclic on $c_{\infty}(\mathbb{Z})=C(\hat{\mathbb{Z}})$ by Theorem 6 , since $\hat{\mathbb{Z}}$ is compact and $B$ is a composition operator associated to the symbol $\varphi: \mathbb{Z} \rightarrow \mathbb{Z}, j \mapsto j+1$.

In the next proposition we show that $\tau_{p}$-supercyclicity does not imply cyclicity for weighted composition operators. Moreover, we prove that cyclicity together with $\tau_{p^{-}}$ supercyclicity do not imply weak supercyclicity. First, consider the spaces

$$
\begin{gathered}
\ell_{\infty}=\left\{f=\left(f_{n}\right)_{n \in \mathbb{N}}, f_{n} \in \mathbb{C}, n \in \mathbb{N}: \sup _{n \in \mathbb{N}}\left|f_{n}\right|<\infty\right\}, \\
c_{\infty}=\left\{f=\left(f_{n}\right)_{n \in \mathbb{N}} \in \ell_{\infty}: \text { is convergent as } n \rightarrow \infty\right\}
\end{gathered}
$$

and

$$
c_{0}=\left\{f=\left(f_{n}\right)_{n \in \mathbb{N}} \in c_{\infty}: \lim _{n \rightarrow \infty} f_{n}=0\right\} .
$$

Observe that $c_{\infty}=C(\hat{\mathbb{N}})$, where $\hat{\mathbb{N}}$ is the Alexandroff compactification of $\mathbb{N}$ and $c_{0}=$ $\operatorname{Ker}\left(\delta_{\infty}\right), \delta_{\infty}: C(\hat{\mathbb{N}}) \rightarrow \mathbb{C}, f \mapsto f(\infty)$. Thus, $c_{0}$ has codimension 1 in $c_{\infty}$. 
The unilateral weighted backward shift $B_{w}: \ell_{\infty} \rightarrow \ell_{\infty}, w=\left(w_{n}\right)_{n \in \mathbb{N}}, w_{n}>0, \lim _{n} w_{n} \rightarrow$ 0 defined by $B_{w} e_{j}=w_{j} e_{j-1}, j=1,2, \ldots, B_{w} e_{0}=e_{0}$, where $e_{0}$ is the zero sequence, can be viewed as a weighted composition operator with symbol $\varphi: \mathbb{N} \rightarrow \mathbb{N}, j \mapsto j+1$, and weight $w=\left(w_{n}\right)_{n \in \mathbb{N}}$. By [25] we know that $B_{w}: c_{0} \rightarrow c_{0}$ is supercyclic.

Lemma 18 Consider a Banach space $G$ and a normed closed subspace $G_{0} \subseteq G$, dense with respect to the pointwise convergence topology $\tau_{p}$. If the codimension of $G_{0}$ in $G$ is greater than 1 and $T: G_{0} \rightarrow G_{0}$ admits a continuous extension $\hat{T}: G \rightarrow G$ such that $\hat{T}(G) \subseteq G_{0}$, then $\hat{T}$ is not cyclic.

Proof. Let $f \in G$ be a cyclic vector of $\hat{T}$. Since the codimension of $G_{0}$ in $G$ is greater than 1 , there exists $g \in G \backslash\left(\operatorname{span}\{f\} \oplus G_{0}\right)$, and therefore, $g \notin \overline{\operatorname{span}\left\{\hat{T}^{n} f: f \geq 0\right\}} \subseteq \operatorname{span}\{f\} \oplus G_{0}$, a contradiction.

Proposition 19 Consider the weight $w=\left(w_{n}\right)_{n}$ such that $w_{n}>0$ and $\lim _{n} w_{n} \rightarrow 0$.

(i) If $f \in \ell_{\infty} \backslash c_{\infty}$, the weighted backward shift $B_{w}: \operatorname{span}\{f\} \oplus c_{\infty} \rightarrow \operatorname{span}\{f\} \oplus c_{\infty}$ is $\tau_{p}$-supercyclic but not (weakly super)cyclic.

(ii) If $f$ is a cyclic vector of $B_{w}: c_{0} \rightarrow c_{0}$ and there exists $g \in c_{\infty}$ such that $B_{w} g=f$, then $B_{w}: c_{\infty} \rightarrow c_{\infty}$ is cyclic, $\tau_{p}$-supercyclic but not weakly supercyclic.

Proof. By [25] it follows that $B_{w}: c_{0} \rightarrow c_{0}$ is supercyclic. Thus, since $c_{0}$ is dense in $\ell_{\infty}$ with respect to the pointwise convergence topology, $B_{w}$ is $\tau_{p}$-supercyclic on $\ell_{\infty}$, on $\operatorname{span}\{f\} \oplus c_{\infty}$ and on $c_{\infty}$.

(i) Since $c_{0}$ has codimension 1 in $c_{\infty}, c_{0}$ has codimension 2 in $\operatorname{span}\{f\} \oplus c_{\infty}$. Moreover, as $\lim _{n} w_{n} \rightarrow 0, B_{w}\left(\operatorname{span}\{f\} \oplus c_{\infty}\right) \subseteq c_{0}$ and thus, $B_{w}: \operatorname{span}\{f\} \oplus c_{\infty} \rightarrow \operatorname{span}\{f\} \oplus c_{\infty}$ is not cyclic by Lemma 18 .

(ii) If $f \in c_{0}$ is a cyclic vector of $B_{w}: c_{0} \rightarrow c_{0}$ and $g \in c_{\infty} \backslash c_{0}$ is such that $B_{w} g=f$, then $c_{\infty}=\operatorname{span}\{g\} \oplus c_{0}$ and $c_{0}=\overline{\operatorname{span}\left\{B_{w}^{n} g: n \geq 1\right\}}$, and so, $B_{w}: c_{\infty} \rightarrow c_{\infty}$ is cyclic. On the other hand, since $B_{w}$ is a weighted composition operator and $c_{\infty}$ is the space $C(\hat{\mathbb{N}})$, where $\hat{\mathbb{N}}$ is the Alexandroff compactification of $\mathbb{N}$, it cannot be weakly supercyclic by Theorem 6 .

Finally, the next example shows that cyclicity does not imply $\tau_{p}$-supercyclicity.

Example 20 The multiplication operator $M_{z} f=z f$ is cyclic but it is not $\tau_{p}$-supercyclic on the disc algebra $A(\mathbb{D})$. Indeed, $\operatorname{span}\left\{\operatorname{Orb}\left(M_{z}, 1\right)\right\}=\operatorname{span}\left\{1, z, z^{2}, \ldots\right\}$ is dense in $A(\mathbb{D})$, but the operator is not weakly supercyclic by $\left[6\right.$, Proposition 1.26] because $\left\{\delta_{z}, z \in \mathbb{D}\right\}$ is a set of independent eigenvectors of $M_{z}^{*}$ in $A(\mathbb{D})^{*}$.

The next diagram illustrates the relations between weak supercyclicity, $\tau_{p}$-supercyclicity and cyclicity for operators.

We now analyze $\tau_{p}$-supercyclicity of $C_{w, \varphi}$ when $X$ is perfect. We first consider $X=\overline{\mathbb{D}}$. Our first result follows as an inmediate consequence of Theorem 8 (v): 


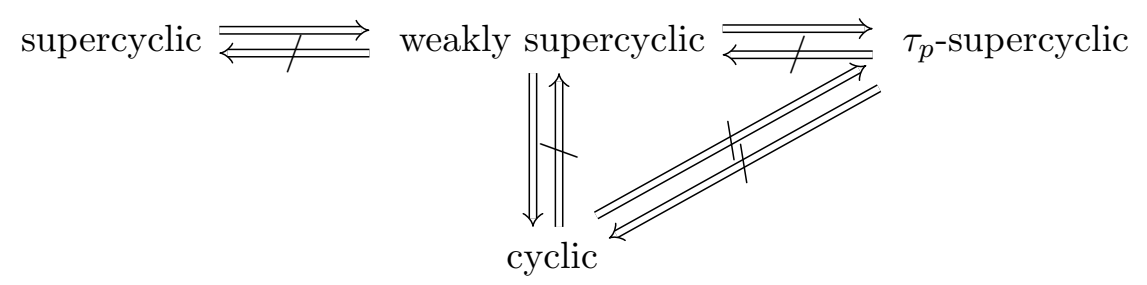

Corollary 21 Let $\lambda \in \mathbb{C}$ with $|\lambda| \leq 1$ and let $\varphi(z)=\lambda z$, then $C_{w, \varphi}: C(\overline{\mathbb{D}}) \rightarrow C(\overline{\mathbb{D}})$ is not $\tau_{p}$-supercyclic.

We now consider a special class of weighted compositions operators and we prove that there are no $\tau_{p}$-supercyclic isometries in the space $C(\overline{\mathbb{D}})$.

Theorem 22 If $T: C(\overline{\mathbb{D}}) \rightarrow C(\overline{\mathbb{D}})$ is a surjective isometry, then it cannot be $\tau_{p}$-supercyclic.

Proof. Let $T: C(\overline{\mathbb{D}}) \rightarrow C(\overline{\mathbb{D}})$ be a surjective isometry. From the Banach-Stone Theorem [19], there exist a homeomorphism $\varphi: \overline{\mathbb{D}} \rightarrow \overline{\mathbb{D}}$ and $w: \overline{\mathbb{D}} \rightarrow \mathbb{C}$ such that $|w(z)|=1$ for all $z \in \overline{\mathbb{D}}$ and $T=C_{w, \varphi}$. Since $\overline{\mathbb{D}}$ is convex, by the Brouwer fixed-point theorem, $\varphi$ has a fixed point $z_{0} \in \overline{\mathbb{D}}$. Since all the evaluations are linearly independent in $(C(\bar{D}))_{\tau_{p}}$ we conclude that $T$ cannot be $\tau_{p}$-supercyclic.

We now analyze $\tau_{p}$-supercyclicity of weighted composition operators acting on $C(\partial \mathbb{D})$, the space of continuous functions on the unit circle. By Proposition 2(ii) and the next Lemma it is enough to consider that the symbol $\varphi: \partial \mathbb{D} \rightarrow \partial \mathbb{D}$ is a homeomorphism.

Lemma 23 If $\varphi: \partial \mathbb{D} \rightarrow \partial \mathbb{D}$ is continuous and injective, then $\varphi$ is a homeomorphism.

Proof. Suppose there exists $\varphi: \partial \mathbb{D} \rightarrow \partial \mathbb{D}$ continuous and injective such that $\varphi(\partial \mathbb{D}) \neq \partial \mathbb{D}$. Since $\varphi$ is continuous, $\varphi(\partial \mathbb{D})$ is compact and connected. Let $t$ be an interior point of $\varphi(\partial \mathbb{D})$ and consider $z \in \partial \mathbb{D}$ such that $t=\varphi(z)$. Since $\varphi$ is injective and not surjective, $\varphi(\partial \mathbb{D} \backslash\{z\})=\varphi(\partial \mathbb{D}) \backslash\{t\}$ is not connected, but $\partial \mathbb{D} \backslash\{z\}$ is so, a contradiction.

In the next propositions we show that $C_{w, \varphi}$ is not $\tau_{p}$-supercyclic on $C(\partial \mathbb{D})$ when $\varphi$ has periodic points or it is a rotation and $|w|=1$.

Proposition 24 Let $\varphi: \partial \mathbb{D} \rightarrow \partial \mathbb{D}$ be a homeomorphism with a periodic point. The weighted composition operator $C_{w, \varphi}: C(\partial \mathbb{D}) \rightarrow C(\partial \mathbb{D})$ is not $\tau_{p}$-supercyclic.

Proof. The dynamics in the case with a single fixed point $z_{0} \in \partial \mathbb{D}$ reduces to the dynamics of continuous functions $f$ on the interval $[0,1]$ that are invertible, with $f(0)=0, f(1)=1$ and $f(x) \neq x$ for every $x \in(0,1)$ [20, Section 2]. Since $f(x)-x$ has constant sign, we can suppose w.l.o.g. that $f(x)-x>0$, and then it follows that $x<\cdots<f^{n-1}(x)<f^{n}(x)<1$ for all $x \in(0,1)$. As a consequence, we get that the iterates must converge to a fixed point, that is, $\lim _{n \rightarrow \infty} f^{n}(x)=1$, and then $\varphi^{n}(z)$ converges to $z_{0}$ for every $z \in \partial \mathbb{D} \backslash\left\{z_{0}\right\}$. By 
Theorem 8 (ii), $C_{w, \varphi}$ is not $\tau_{p}$-supercyclic. In the case $\varphi$ has a periodic non fixed point Theorem 8 (i) and (iii) yield the result.

Proposition 25 Let $\varphi: \partial \mathbb{D} \rightarrow \partial \mathbb{D}, \varphi(z)=\lambda z$ for some $\lambda \in \mathbb{C}$ with $|\lambda|=1$ and w such that $|w|=1$. The operator $C_{w, \varphi}: C(\partial \mathbb{D}) \rightarrow C(\partial \mathbb{D})$ is never $\tau_{p}$-supercyclic.

Proof. Assume that $C_{w, \varphi}$ is $\tau_{p}$-supercyclic. Let $f$ be a $\tau_{p}$-supercyclic function such that $\|f\|_{\infty}=1$. Given $g \in C(\partial \mathbb{D})$ such that $\|g\|_{\infty}=1$, there exists a net such that $\left(\alpha_{i} C_{\varphi, w}^{n(i)} f\right)_{i \in I} \rightarrow g$ in $\tau_{p}$, and thus, $\left(\left|\alpha_{i}\right|\left|f\left(\varphi^{n(i)}\right)\right|\right)_{i \in I} \rightarrow|g|$ in $\tau_{p}$. Since $\partial \mathbb{D}$ is compact, by extracting a subnet, we can assume w.l.o.g. that $\lambda^{n(i)} \rightarrow \lambda_{0}$ for some $\lambda_{0} \in \partial \mathbb{D}$. Let $z_{0} \in \partial \mathbb{D}$ such that $\left|f\left(\lambda_{0} z_{0}\right)\right|=1$. Since $\left|f\left(\varphi^{n(i)}\left(z_{0}\right)\right)\right| \rightarrow\left|f\left(\lambda_{0} z_{0}\right)\right|=1$ and $\left|f\left(\varphi^{n(i)}\left(z_{0}\right)\right)\right| \leq 1$, there exists $i_{0}$ such that $\left|f\left(\varphi^{n(i)}\left(z_{0}\right)\right)\right| \in[1 / 2,1]$ and

$$
\left|\alpha_{i}\right|\left|f\left(\varphi^{n(i)}\left(z_{0}\right)\right)\right|-\left|g\left(z_{0}\right)\right|<1 \text {, i.e., }\left|\alpha_{i}\right|\left|f\left(\varphi^{n(i)}\left(z_{0}\right)\right)\right|<2
$$

for every $i \geq i_{0}$. Hence, $\left|\alpha_{i}\right| \leq \frac{2}{\left|f\left(\varphi^{n(i)}\left(z_{0}\right)\right)\right|} \leq 4$. Let $\left(\left|\alpha_{j}\right|\left|f\left(\varphi^{n(j)}\right)\right|\right)_{j \in J}, J \subseteq I$, be a subnet such that $\left|\alpha_{j}\right|_{j \in J}$ converges to $\alpha \geq 0$. Now, $\left(\left|\alpha_{j}\right|\left|f\left(\varphi^{n(j)}\right)\right|\right)_{j \in J} \rightarrow \alpha\left|f\left(\lambda_{0} \cdot\right)\right|$ in $\tau_{p}$ and also $\left(\left|\alpha_{j}\right|\left|f\left(\varphi^{n(j)}\right)\right|\right)_{j \in J} \rightarrow|g|$. Hence, $\alpha=1$ and $|g(z)|=\left|f\left(\lambda_{0} z\right)\right|$ for every $z \in \partial \mathbb{D}$. Therefore, it follows that $\|g\|_{L^{1}}=\|f\|_{L^{1}}$ for every $g \in B_{C(\partial \mathbb{D})}$, a contradiction.

Given an interval $I$ and $\varphi: I \rightarrow I$, the $\omega$-limit set of the orbit of $x \in I$ is the set

$$
\omega(x)=\left\{y \in I: \exists n_{k} \text { with } \lim _{k \rightarrow \infty} \varphi^{n_{k}}(x)=y\right\} .
$$

We say that $\varphi: I \rightarrow I$ has a wandering interval $J$ if the intervals $J, \varphi(J), \varphi^{2}(J), \ldots$ are pairwise disjoint and the $\omega$-limit set of $J$ is not equal to a single periodic orbit. Examples of symbols $\varphi: \partial \mathbb{D} \rightarrow \partial \mathbb{D}$ with non-wandering intervals are the $C^{2}$ diffeomorphisms without periodic points, the $C^{1}$ diffeomorphisms whose derivative is a function of bounded variation or the analytic homeomorphisms of the circle without periodic points [40, Chapter 1 , Section 2].

Theorem 26 If $\varphi: \partial \mathbb{D} \rightarrow \partial \mathbb{D}$ does not have a wandering interval $J$ and $|w|=1$, then the weighted composition operator $C_{w, \varphi}: C(\partial \mathbb{D}) \rightarrow C(\partial \mathbb{D})$ is not $\tau_{p}$-supercyclic.

Proof. Assume $\varphi: \partial \mathbb{D} \rightarrow \partial \mathbb{D}$ does not have a wandering interval $J$ and has no periodic points (otherwise, apply Proposition 24). By [40, page 36], $\varphi$ is conjugate to a rotation $\tilde{\varphi}$, i.e., there exists a homeomorphism $h: \partial \mathbb{D} \rightarrow \partial \mathbb{D}$ such that $\tilde{\varphi}=h^{-1} \circ \varphi \circ h$, and thus, $C_{w, \varphi}$ is similar to $C_{w \circ h, \tilde{\varphi}}[23]$. Since $C_{w \circ h, \tilde{\varphi}}$ is not $\tau_{p}$-supercyclic by Proposition 25, then $C_{w, \varphi}$ is not $\tau_{p}$-supercyclic $[29]$.

From all our results it seems natural to conjecture that if $X$ is compact, $E \hookrightarrow\left(C(X), \tau_{p}\right)$ is a Banach space and the operator $C_{w, \varphi}: E \rightarrow E$ is isometric, then $C_{w, \varphi}$ is not $\tau_{p^{-}}$ supercyclic. 


\section{Acknowledgments}

The authors are very thankful to the referee for his/her careful reading of the manuscript and his/her valuable comments and observations.

The first and the second author were supported by MEC, MTM2016-76647-P. The third author was supported by MEC, MTM2016-75963-P and GVA/2018/110.

\section{References}

[1] A. Albanese, D. Jornet, A note on supercyclic operators in locally convex spaces Mediterr. J. Math. 16 (2019), no. 5, Art. 107, 10 pp.

[2] A. Aleman, L. Suciu, On ergodic operator means in Banach spaces. Integr. Equ. Oper. Theory 85(2) (2016), 259-287.

[3] S. Ansari, Hypercyclic and cyclic vectors. J. Funct. Anal. 128(2) (1995), 374-383.

[4] S. I. Ansari, P. S. Bourdon, Some properties of cyclic operators, Acta Sci. Math. 63 (1997), 195-207.

[5] F. Bayart, É. Matheron, Hyponormal operators, weighted shifts and weak forms of supercyclicity. Proc. Edinb. Math. Soc. 49 (2006), 1-15.

[6] F. Bayart, É. Matheron, Dynamics of linear operators, Cambridge University Press, Cambridge, 2009.

[7] S. Bermudo, A. Montes-Rodríguez, S. Shkarin, Orbits of operators commuting with the Volterra operator. J. Math. Pures Appl. 89(2) (2008), 145-173.

[8] L. Bernal-Rodríguez, A. Montes-Rodríguez, Universal functions for composition operators. Complex Variables Theory Appl. 27 (1995), no. 1, 4756.

[9] J. Bès, Dynamics of weighted composition operators, Complex Anal. Oper. Theory 8 (2014), 159-176.

[10] J. Bonet, P. Domański, Hypercyclic operators on spaces of real analytic functions, Math. Proc. Cambridge Philos. Soc. 153(3) (2012), 489-503.

[11] J. Bonet, A. Peris, Hypercyclic operators on non-normable Fréchet spaces, J. Funct. Anal. 159 (1998), 587-595.

[12] P. S. Bourdon, J. S. Shapiro, Cyclic Phenomena for Composition Operators, Mem. Amer. Math. Soc. 125 (1997), no. 596, Providence, Rhode Island.

[13] K. C. Chan, R. Sanders, A weakly hypercyclic operator that is not norm hypercyclic, Journal of Operator Theory (2004), 39-59. 
[14] B. P. Duggal, Weak supercyclicity: dynamics of paranormal operators. Rend. Circ. Mat. Palermo 65(2) (2016), 297-306.

[15] C. Fernández, A. Galbis, E. Jordá, Dynamics and spectra of composition operators on the Schwartz space. J. Funct. Anal. 274(12) (2018), 3503-3530.

[16] K.G. Grosse-Erdmann, R. Mortini, Universal functions for composition operators with non-automorphic symbol, J. Anal. Math. 107 (2009), 355-376.

[17] C. Cowen, B. MacCluer, Composition Operators on Spaces of Analytic Functions, Studies in Advanced Mathematics. CRC Press, Boca Raton, FL, 1995.

[18] D. J. H. Garling, A course in Mathematical analysis: Volume III, Complex analysis, measure and integration. Cambridge University Press, New York, 2013.

[19] M. I. Garrido, J. A. Jaramillo, Variations on the Banach-Stone theorem. In IV Course on Banach spaces and Operators (Laredo, 2001), Extracta Math. 17 (2002), 351-383.

[20] S. Gadgil, Dynamics on the Circle-Interval Dynamics and Rotation Number. Resonance Journal of Science Education 8(11) (2003), 25-36

[21] K. G. Grosse-Erdmann, A. Peris, Linear Chaos. Springer, Berlin, 2011.

[22] K. G. Grosse-Erdmann, R. Mortini, Universal functions for composition operators with non-automorphic symbol. J. Anal. Math. 107 (2009), 355-376.

[23] G. Gunatillake, Invertible weighted composition operators, J. Funct. Anal. 261 (2011), $831-860$.

[24] D. A. Herrero, Limits of hypercyclic and supercyclic operators. J. Funct. Anal. 99(1) (1991), 179-190.

[25] H. M. Hilden, L. J. Wallen, Some cyclic and non-cyclic vectors of certain operators, Indiana Univ. Math. J. 23 (1974), 557-565.

[26] T. Kalmes, Dynamics of weighted composition operators on function spaces defined by local properties, Studia Math. 249 (2019), no. 3, 259-301.

[27] Z. Kamali, K. Hedayatian, B. Khani Robati, Non-weakly supercyclic weighted composition operators. Abstr. Appl. Anal. Art. (2010) ID 143808.

[28] G. Köthe, Topological Vector Spaces II. Springer, New York, 1979.

[29] Y. X. Liang, Z. H. Zhou, Supercyclic tuples of the adjoint weighted composition operators on Hilbert spaces, Bull. Iranian Math. Soc. 41(1) (2015), 121-139.

[30] J. Milnor, Dynamics in One Complex Variable. Third Edition. Princeton University Press, Princeton, 2006. 
[31] A. Montes-Rodríguez, A. Rodríguez-Martínez, S. Shkarin, Cyclic behaviour of Volterra composition operators. Proc. Lond. Math. Soc. 103(3) (2011), 535-562.

[32] A. Montes-Rodríguez, S. Shkarin. Non-weakly supercyclic operators. J. Operator Theory 58(1) (2007), 39-62.

[33] A. Moradi, B. Khani Robati, K. Hedayatian, Non-Weakly Supercyclic Classes of Weighted Composition Operators on Banach Spaces of Analytic Functions, Bull. Belg. Math. Soc. Simon Stevin 24(2) (2017), 227-241.

[34] A. Peris, Multi-hypercyclic operators are hypercyclic. Math. Z. 236(4) (2001), 779-786.

[35] R. Sanders, Weakly supercyclic operators, J. Math. Anal. Appl. 292 (2004) 148-159.

[36] R. Sanders, An Isometric Bilateral Shift that is Weakly Supercyclic, Integr. Equ. Oper. Theory 53 (2005), 547-552.

[37] J. H. Shapiro, Composition operators and classical function theory. Universitext: Tracts in Mathematics. Springer-Verlag, New York, 1993.

[38] J. H. Shapiro, Simple connectivity and linear chaos. Rend. Circ. Mat. Palermo (2) Suppl 56 (1998), 27-48.

[39] S. Shkarin, Non-sequential weak supercyclicity and hypercyclicity. J. Funct. Anal. 242(1) (2007), 37-77.

[40] M. de Welington, S. van Strien, One-Dimensional Dynamics, Springer-Verlag Berlin Heidelberg (1993).

[41] B. Yousefi, H. Rezaei, Hypercyclic property of weighted composition operators, Proc. Amer. Math. Soc. 135(10) (2007), 3263-3271. 